World Scientific Journals will be set by the publisher

DOI: will be set by the publisher

(c) Owned by the authors, published by EDP Sciences, 2019

\title{
Measurement of cross-sections and Properties of the Higgs Bo- son using the ATLAS Detector
}

\author{
Luigi Marchese ${ }^{1, a}$ on behalf of the ATLAS Collaboration \\ ${ }^{1}$ University of Oxford
}

\begin{abstract}
In this paper we review the latest measurements of the Higgs boson production and decay performed at the Large Hadron Collider by the ATLAS experiments. These measurements are based on $\sqrt{s}=13 \mathrm{TeV}$, corresponding to integrated luminosities ranging from 36.1 to $79.8 \mathrm{fb}^{-1}$. This rich dataset allows for the observation of the associated production of the Higgs boson with a $t \bar{t}$ pair, the vector-boson-associated production of the Higgs boson, $V H$, and the observation of the Higgs boson decay to a pair of $b \bar{b}$ quarks. Analyses in both fermionic and bosonic decay channels are discussed. A final combination on the Higgs boson coupling properties is summarized.
\end{abstract}

\section{Introduction}

After the ATLAS [1] and CMS experiments of the Large Hadron Collider (LHC) observed a particle consistent with the Standard Model (SM) Higgs boson $(H)$ in 2012 [2] [3] , a broad range of properties of this particle were studied using proton-proton collision collected at center-of-mass energies of $\sqrt{s}=$ 7 and 8 TeV during Run 1(2011-2012). Measured results are found to be consistent with the Standard Model expectations. The mass of this boson was determined to be approximately $125 \mathrm{GeV}$ with a $0.2 \%$ accuracy [4], the $0^{+}$spin-parity assignment was established [5] [6] and its measured couplings to SM particles were found to be consistent with the expected predictions with accuracies around $10 \%$ [7]. The Run 1 dataset allowed only for the observation of the gluon gluon fusion $(g g F)$ and vector boson fusion $(V B F)$ production modes. Only decays to vector bosons were observed, while only when combining the ATLAS and CMS results it was possible to observe the Higgs decay to a pair of $\tau$ leptons.

In 2015 the LHC started the Run-2 data taking with the upgrade of the center-of-mass energy to $\sqrt{s}=13 \mathrm{TeV}$ and an increased instantaneous luminosity of up to $2 \cdot 10^{34} \mathrm{~cm}^{-2} \mathrm{~s}^{-1}$, exceeding by a factor of 2 the LHC design luminosity. Results presented in this paper are based on either an integrated luminosity around $36 \mathrm{fb}^{-1}$, data collected in 2015-2016, or on an integrated luminosity around $80 \mathrm{fb}^{-1}$, data collected in 2015-2017. The discovery of the $t \bar{t} H$ and $V H$ production modes, the observation of the decay $H \rightarrow b \bar{b}$ and new precision measurements of the Higgs properties in the bosonic channels $\left(H \rightarrow Z Z^{*}, H \rightarrow W W^{*}\right.$ and $H \rightarrow \gamma \gamma$ ) were finally possible when exploiting this rich sample.

At the LHC, the Higgs boson is mainly produced via the gluon gluon fusion process, $g g F$, mediated by a top quark loop ( $88 \%)$, the vector boson fusion process, $V B F$, with a clear signature of

ae-mail: luigi.marchese@cern.ch 
two scattered quarks in addition to the produced Higgs boson (7\%), the vector-boson-associated production with a vector boson $W$ or $Z, V H,(4 \%)$ and finally the associated production of the Higgs boson with a $t \bar{t}$ pair, $t \bar{t} H,(1 \%)$. Among the various decay channels, the most abundant are $b \bar{b}(58 \%)$, $W W^{*}(21 \%), g g(8.2 \%), \tau \tau(6.2 \%), c \bar{c}(2.9 \%), Z Z^{*}(2.6 \%), \gamma \gamma(0.2 \%), Z \gamma(0.15 \%)$ and $\mu \mu(0.02 \%)$.

This paper is structured as follows: Section 2 describes the latest Run-2 observations; Section 3 describes results in the leptonic decays; Section 4 describes results and properties measured in the bosonic decays and Section 5 presents the combination of the Higgs boson couplings properties. Finally, in Section 6 we summarize the various results introduced in the previous sections.

\section{Run-2 Observations}

In Run-2 we finally observed the missing production modes, $t \bar{t} H$ and $V H$. We also observed the Higgs boson decay to a pair of $b \bar{b}$ quarks.

\section{$2.1 t \bar{t} H$ production}

Higgs couplings to the top quark, the heaviest SM particle, were indirectly probed in Run 1 by studying the ggF production mode assuming the main loop contribution is from top quarks. The $t \bar{t} H$ production mode provides a direct channel to probe such coupling. Although we quadruple the $t \bar{t} H$ production cross section when compared to the corresponding value at the Run-1-center-of-mass energy, this channel remains experimentally challenging because of the complex final states. In fact, this production mode leads to a final state with $b b W W$ arising from the top quark decays in addition to the Higgs boson decay products. Different analyses are performed to target the various Higgs decay channels: $t \bar{t} H(\rightarrow \gamma \gamma), t \bar{t} H\left(\rightarrow Z Z^{*} \rightarrow 4 \ell\right), t \bar{t} H(\rightarrow b \bar{b})$ and $t \bar{t} H\left(\rightarrow W W^{*}\right.$ or $\tau \tau$ with multilepton final states). Multivariate strategies and techniques have been implemented in the various analyses to enhance the signal sensitivity. Depending on the channel, an integrated luminosity of $36.1 \mathrm{fb}^{-1}$ $(t \bar{t} H$ multilepton and $t \bar{t} H(\rightarrow b \bar{b}))$ and of $79.8 \mathrm{fb}^{-1}(t \bar{t} H(\rightarrow \gamma \gamma))$ has been used. The most stringent results are from the $t \bar{t} H$-multilepton and $t \bar{t} H(\rightarrow \gamma \gamma)$ channels leading to an observed(expected) significance of 4.1(3.7) $\sigma$ [8] and 4.1(2.8) $\sigma$ [9], respectively. Although the $t \bar{t} H\left(\rightarrow Z Z^{*} \rightarrow 4 \ell\right)$ channel has the cleanest signature, the expected low rate of $<1$ event makes it the least sensitive of all the decay channels.

Combining the various channels and the corresponding Run-1 results, the observed(expected) significance for the $t \bar{t} H$ production mode is 6.3(5.1) $\sigma$ [9] and the measured signal strength $\mu$ is:

$$
\mu=1.32 \pm 0.18(\text { stat. })_{-0.19}^{+0.21}(\text { syst. })
$$

In Fig.1(left), the combined mass distribution of one of the most sensitive channels, $t \bar{t} H(\rightarrow \gamma \gamma)$, is shown. The mass peak is visible above the background. In Fig.1(right) we show the measured signal strengths in all the $t \bar{t} H$ analyses along with their combination. The SM compatibility is within $2 \sigma$.

\section{$2.2 V H$ production and $H \rightarrow b \bar{b}$}

Despite the large branching ratio for $H \rightarrow b \bar{b}$ (58\%), it was possible to observe it only in Run 2 . It's experimentally challenging to identify $b$-jets arising from the Higgs decay due to the abundant background with hadronic signatures. The $V H$ production mode provides the most sensitive channel to observe $H \rightarrow b \bar{b}$ since the leptonic decays of the $W / Z$ bosons suppress most of the multi-jet background. However, at high Higgs boson transverse momentum the $t \bar{t} H, g g F$ and $V B F$ production 

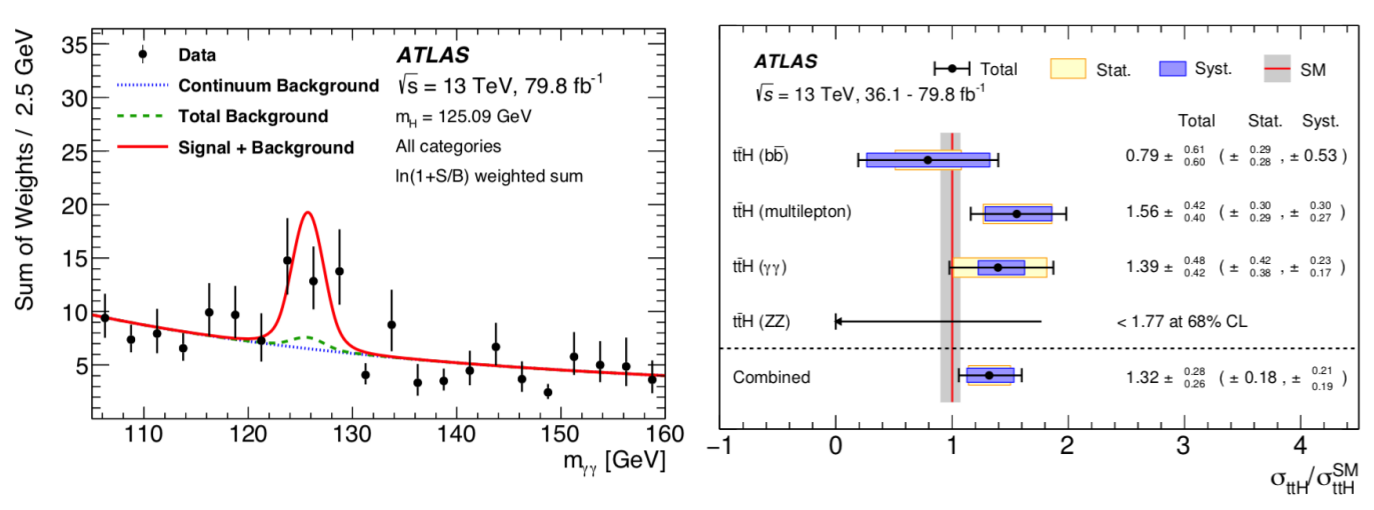

Figure 1: Combined mass distribution of one of the most sensitive channels, $t \bar{t} H(\rightarrow \gamma \gamma)$ [9] (left); combined measured signal strengths of the various $t \bar{t} H$ analyses [9] (right).

modes are also investigated [10]. All the three analyses make use of multivariate techniques to increase the signal-to-background ratio exploiting some peculiar features of $b$-hadrons when compared to lighter hadrons such as lifetime differences and jet information. Due to the long $b$-hadrons lifetime, it's possible to clearly resolve production and decay vertices and identify charged decay products characterized by a large impact parameter. The $V H$ analysis is split into three categories depending on the number of leptons (electrons and muons) in the final state: events with 0 leptons with missing transverse momentum and 2 leptons target the associated Higgs production with a $Z$ boson decaying to a pair of $v$ or 2 leptons, respectively; events with 1 lepton target the associated Higgs production with a $W(\rightarrow \ell v)$ boson. Two $b$-tagged jets are required. Crucial variables to separate signal and background are the $b \bar{b}$ invariant mass and other kinematical variables like the $p_{T}$ of the vector boson. The signal sensitivity is further enhanced by introducing a multivariate discriminant trained to properly separate signal and background in the different decay categories. The analysis strategy is validated studying the search for the $V Z$ production followed by $Z \rightarrow b \bar{b}$ decay, a topology very similar to the Higgs signal except for the lower $b \bar{b}$ invariant mass. The observed(expected) sensitivity when combining the 79.8 $\mathrm{fb}^{-1}$ of $13 \mathrm{TeV}$ with the Run-1 similar searches for the $V H$ production mode in the various decays channels is 5.3(4.8) $\sigma$ [11] establishing the $V H$ observation at LHC with a measured signal strength $\mu=1.13 \pm 0.15$ (stat. $)_{-01.7}^{+0.18}$ (syst.). The combination of $H \rightarrow b \bar{b}$ produced via the $V H$ production mode with similar measurements via the $V B F$ [12] and $t \bar{t} H$ [13] in both Run 1 and Run 2 yields an observed(expected) significance of 5.4(5.5) $\sigma$ with a signal strength $\mu=1.01 \pm 0.12$ (stat. $)_{-0.15}^{+0.16}($ syst.) [11]. After a long search, the decay channel $H \rightarrow b \bar{b}$ is finally observed and in agreement with the SM.

In Fig. 2 we show the combined and single-analysis results for the $V H$ production mode (left) and the $H \rightarrow b \bar{b}$ decay channel (right).

\section{Leptonic Decays}

In Run 2 new searches and observations were performed in the context of Higgs couplings to fermions. As described in the previous sections, ATLAS observed the $t \bar{t} H$ production mode and the $H \rightarrow b \bar{b}$ decay channel probing Higgs couplings to the top and the beauty quark, respectively. In addition, in 

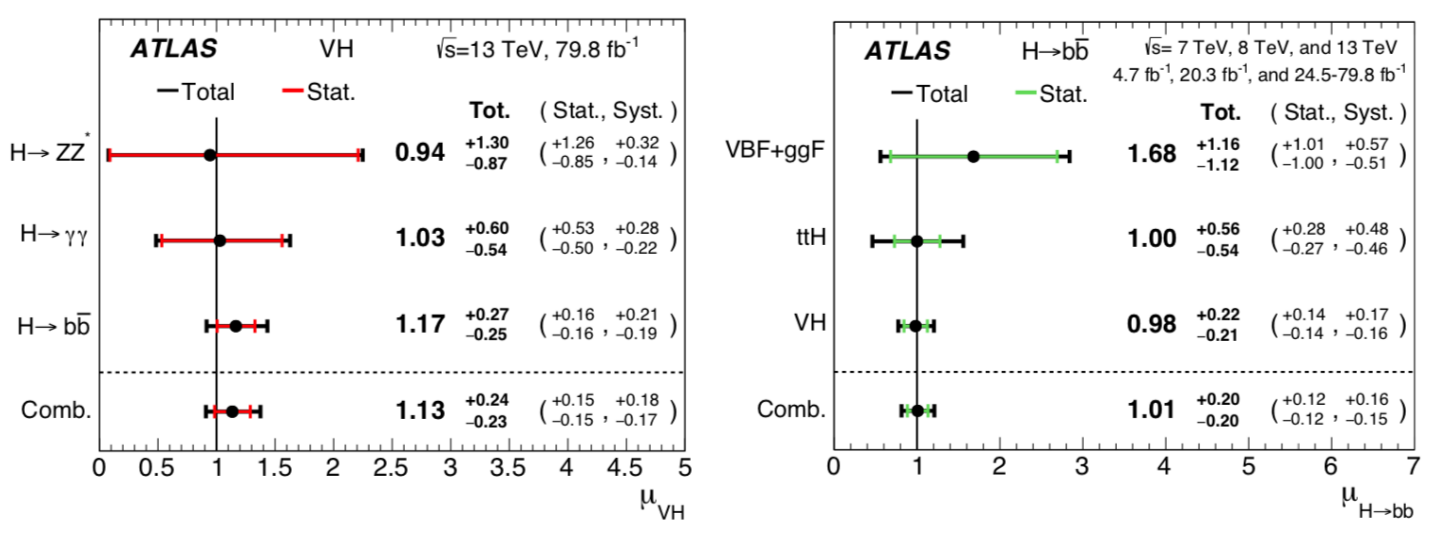

Figure 2: Combined and single-analysis measured signal strengths of the various $V H$ analyses (left) and the various $H \rightarrow b \bar{b}$ analyses [11] (right).

Run 2 ATLAS also observed, independently of CMS, the $H \rightarrow \tau \tau$ decay channel and performed two searches for $H \rightarrow \mu \mu$ and $H \rightarrow c \bar{c}$.

\section{$3.1 H \rightarrow \tau \tau$}

In Run 1 the observation of the Higgs decay channel, $H \rightarrow \tau \tau$, was possible only when combining ATLAS and CMS results. A new search was performed in Run 2 looking at the leptonic or hadronic decay modes of the $\tau$ leptons. The main background, the $Z / \gamma^{*} \rightarrow \tau \tau$ process, is estimated from simulation after introducing a data-based normalization factor. Several categories are considered depending on the Higgs boson production mode. The $V B F$ and the $g g F$-boosted- $H$-production categories are the most sensitive. In each category the signal yield is extracted from fits of the $\tau \tau$ invariant mass distribution. Based on an integrated luminosity of $36.1 \mathrm{fb}^{-1}$, ATLAS was finally able to observe the $H \rightarrow \tau \tau$ decay channel independently of CMS [14]. A precision around $25 \%$ is achieved for the measurement of the total cross section in agreement with the SM expectation.

\section{$3.2 H \rightarrow \mu \mu$ and $H \rightarrow c \bar{c}$}

Starting from the $H \rightarrow b \bar{b}$ analysis, a dedicated $H \rightarrow c \bar{c}$ strategy is developed focusing on specific $c$-jet identification algorithms. The signal-to-background ratio is enhanced by looking at the Higgs associated production with a $Z$ boson, $Z(\rightarrow e e$ or $\mu \mu) H(\rightarrow c \bar{c})$. Based on an integrated luminosity of $36.1 \mathrm{fb}^{-1}$, ATLAS reports an upper limit on the signal strength with respect to the SM expectation of $\mu<110$ at the $95 \%$ confidence level [15].

Assuming that even at the HL-LHC, ATLAS and CMS probably won't be able to observe the $H \rightarrow c \bar{c}$ SM decay channel [16], $H \rightarrow \mu \mu$ will be the only decay channel to probe Higgs couplings to second-generation fermions [17]. Using an integrated luminosity of $79.8 \mathrm{fb}^{-1}$, a search for $H \rightarrow \mu \mu$ was performed in Run 2. The dominant background is the abundant Drell-Yan process. Categories are defined targeting the $g g F$ and $V B F$ production modes making use of multivariate techniques to enhance the signal sensitivity. The observed limit at $95 \%$ confidence level is 2.1 times the SM expectation [18]. 


\section{Bosonic decays}

The main Higgs decays to bosons were all observed in Run 1. $H \rightarrow Z Z^{*}$ and $H \rightarrow \gamma \gamma$ were the Higssboson-discovery channels while $H \rightarrow W W^{*}$ provided the most precise cross-section measurement in Run 1. New measurements have been performed in Run 2.

\section{$4.1 H \rightarrow W W^{*}$}

$H \rightarrow W W^{*}$ is the most abundant bosonic channel (21\%). However, when considering the clearest experimental signature, the dilepton final state $H \rightarrow W W^{*} \rightarrow \ell v \ell v$, the branching ratio is reduced to $1.1 \%$. The $e-\mu$ channel is the most sensitive one. This decay channel is characterized by a low mass resolution since the Higgs boson cannot be reconstructed due to the two undetected neutrinos. Although the signal rate is large, the signal-to-background ratio is smaller than one, even after a stringent kinematic selection. Categories based on the different production mode are introduced. Results are provided separately for the $g g \mathrm{~F}$ and $V B F$ production modes. Using an integrated luminosity of $36.1 \mathrm{fb}^{-1}$, ATLAS measured $\sigma_{g g F} \cdot B R_{H W W^{*}}=12.6_{-1.2}^{+1.3}(\text { stat. })_{-1.8}^{+1.9}($ syst. $) \mathrm{pb}$ and $\mu_{g g F}=1.21_{-0.21}^{+0.22}$ and $\sigma_{V B F} \cdot B R_{H W W^{*}}=0.50_{-0.23}^{+0.24}($ stat. $)+0.18$ (syst. $) \mathrm{pb}$ and $\mu_{V B F}=0.62_{-0.36}^{+0.37}[19]$.

\section{2 $H \rightarrow Z Z^{*}$ and $H \rightarrow \gamma \gamma$}

The decay channels $H \rightarrow Z Z^{*} \rightarrow 4 \ell(e$ or $\mu)$ and $H \rightarrow \gamma \gamma$ have the clearest experimental signature. Having both the channels final state particles (electron, muons or photons) measurable with high experimental resolution, they both have an excellent mass resolution, $O(1 \mathrm{GeV})$. Moreover, the main background (irreducible $Z Z^{*}$ production and continuum $\gamma \gamma$ production, respectively) is well modelled. They were the Higgs-boson-discovery channels in Run 1 and are now used for precisions measurements like the Higgs-boson-mass or differential-cross-section measurements. Using an integrated luminosity of $79.8 \mathrm{fb}^{-1}$, the Run- 2 measured results of the ratio between the observed and predicted yields are $1.19_{-0.15}^{+0.16}$ for $H \rightarrow Z Z^{*}[20]$ and $1.06_{-0.12}^{+0.14}$ [21].

\subsubsection{Precision measurements}

The Higgs boson mass can be measured by performing a fit to the invariant mass of the diphoton pair or the $4 \ell$. Combining the Run-2 results $\left(36.1 \mathrm{fb}^{-1}\right)$ obtained from the two decay channels with the corresponding results from the Run 1, the ATLAS collaboration reports a value of the Higgs boson mass of $124.97 \pm 0.24 \mathrm{GeV}$ [22]. The 1.9 permille precision is achieved using only the ATLAS datasets, while in Run 1 such precision was achieved when combining the ATLAS and CMS results. In Fig. 3 we show the Run- 2 fit to the diphoton and $4 \ell$ invariant mass.

Similarly, the Higgs boson width can be added as a free parameter and extracted from the same fit. However, the esperimental resolution of $O(1 \mathrm{GeV})$ must be convolved with the natural Higgs width expected to be $4 \mathrm{MeV}$ in the SM. As a result, the best limit set with this strategy is $\sim 630$ times larger than the SM expectation. A new indirect model-dependent limit on $\Gamma_{H}$ can be derived by measuring the ratio of the number of $g g \rightarrow H^{*} \rightarrow Z Z$ events in the off-shell Higgs mass region to the number of $g g \rightarrow H \rightarrow Z Z^{*}$ events in the on-shell Higgs mass region. The Run-2 model-dependent constrain on $\Gamma_{H}$ based on an integrated luminosity of $36.1 \mathrm{fb}^{-1}$ is $14.4 \mathrm{MeV}$ at $95 \%$ confidence level [23].

In addition, the $H \rightarrow Z Z^{*}$ and $H \rightarrow \gamma \gamma$ channels can be used to measure the differential crosssections as a function of several variables. New Higgs interactions, not visible in the measurements of the total Higgs boson cross sections, could be observed in the differential cross-section measurements opening a window into new physics. In Fig. 4 we show the differential cross-section as a function 
World Scientific Journals
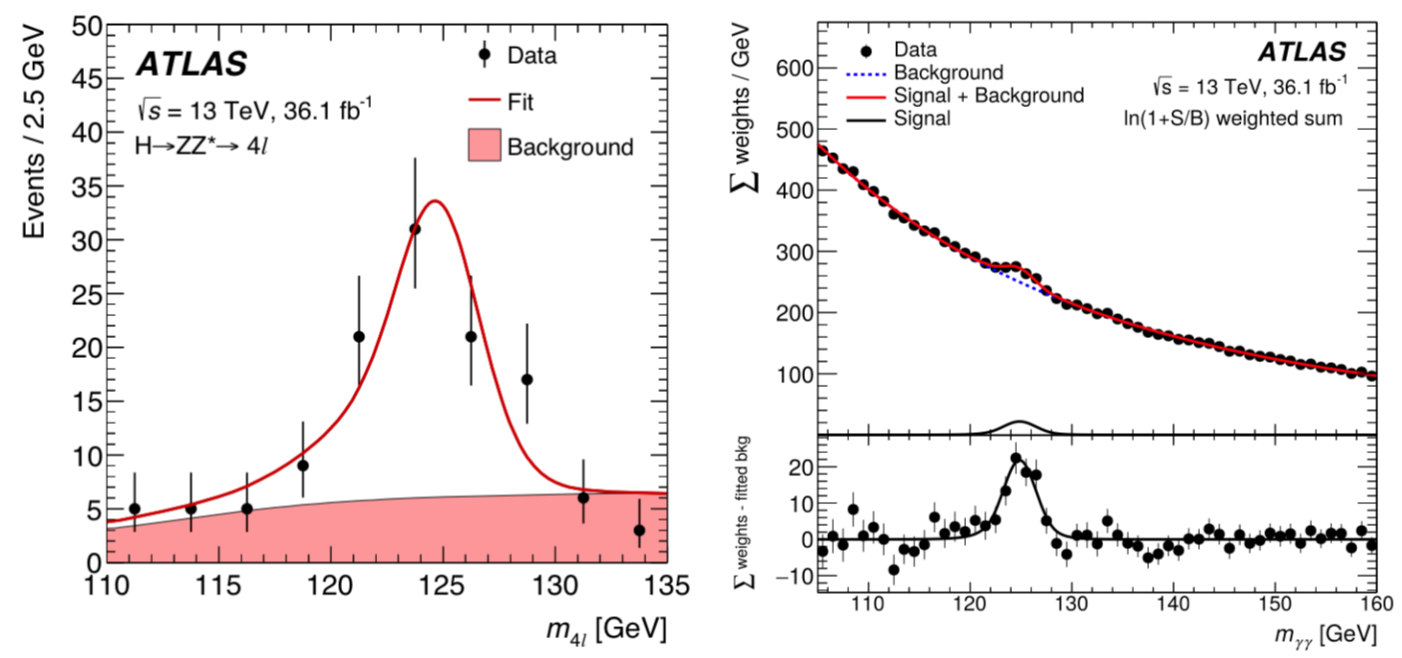

Figure 3: Fit to the $4 \ell$ (left) and diphoton (right) invariant mass to extract the Higgs boson mass [22].

of the Higgs boson transverse momentum for both the channels $H \rightarrow Z Z^{*}$ (left) and $H \rightarrow \gamma \gamma$ (right) using $79.8 \mathrm{fb}^{-1}$. Results agree well within systematic uncertainties with several theoretical predicitons [24] [25] indicating no hints of new physics.
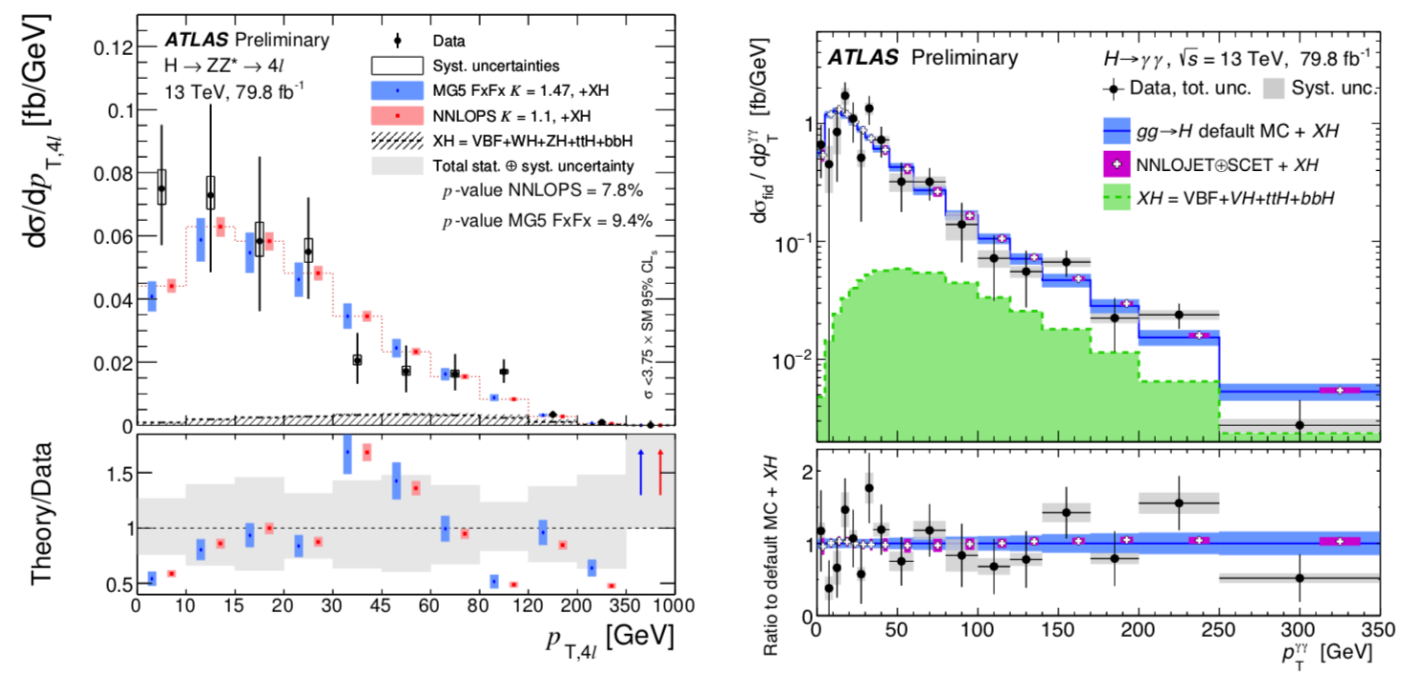

Figure 4: Differential Higgs cross-section as a function of the Higgs boson transverse momentum for both the channels $H \rightarrow Z Z^{*}$ [24] (left) and $H \rightarrow \gamma \gamma$ [25] (right). 


\section{Combination of the Higgs boson results}

The various Run-2 measurements presented in the previous sections for all the Higgs production modes $(g g F, V B F, V H$ and $t \bar{t} H)$ and the various decay channels $(H \rightarrow b \bar{b}, H \rightarrow \tau \tau, H \rightarrow \mu \mu$, $H \rightarrow Z Z^{*}, H \rightarrow W W^{*}$ and $H \rightarrow \gamma \gamma$ ) can be combined leading to a combined Higgs signal strength with respect to the SM expectation of: $\mu=1.13_{-0.08}^{+0.09}$ [26]. In Fig.5 we report the Run-2 measured signal strengths in the various Higgs boson production modes and decay channels (left) and the Run2 measured coupling strengths modifiers as a function of the mass of the particle the Higgs boson couples to. No deviations from the SM expectations are observed.
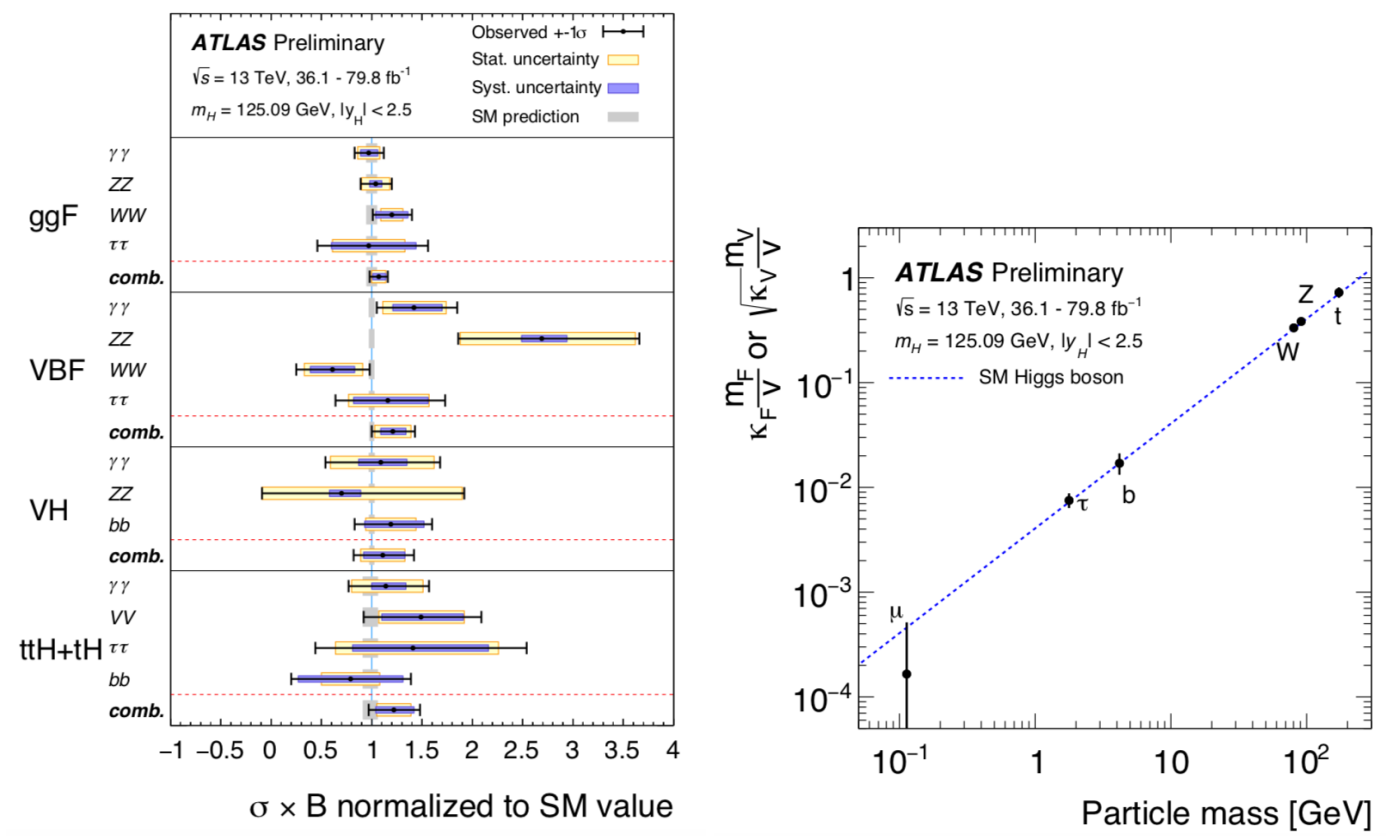

Figure 5: Run-2 measured Higgs boson signal strengths for the various production modes and decay channels (left) and Run-2 measured scaling of the Higgs boson couplings as a function of the particle mass (right) [26].

\section{Conclusion}

The latest Higgs measurements based on 36.1 to $79.8 \mathrm{fb}^{-1}$ of proton-proton collision data recorded at the LHC by the ATLAS experiment at $13 \mathrm{TeV}$ have been reviewed. In Run 2 we finally observed all the four main Higgs production modes ( $g g F, V B F, V H$ and $t \bar{t} H)$ and five Higgs boson decay channels $\left(H \rightarrow b \bar{b}, H \rightarrow \tau \tau, H \rightarrow Z Z^{*}, H \rightarrow W W^{*}\right.$ and $\left.H \rightarrow \gamma \gamma\right)$. In addition, several Higgs precison measurements have been performed exploiting the excellent mass resolution of the $H \rightarrow Z Z^{*}$ and $H \rightarrow \gamma \gamma$ channels obtaining a precision of 1.9 permille on the Higgs boson mass measurement, an indirect model-dependent constrain on the Higgs boson total width of $14.4 \mathrm{MeV}$ and quasi-model independent measurements of differential cross-sections. Results are consistent with the SM expectations indicating no hints of new physics. 
Copyright 2019 CERN for the benefit of the ATLAS Collaboration. Reproduction of this article or parts of it is allowed as specified in the CC-BY-4.0 license.

\section{References}

[1] ATLAS Collaboration, 2008 JINST 3 S08003

[2] ATLAS Collaboration, Phys. Lett. B716 (2012) 1

[3] CMS Collaboration, Phys. Lett. B716 (2012) 30

[4] ATLAS and CMS Collaboration, Phys. Rev. Lett. 114 (2015) 191803

[5] ATLAS Collaboration, Eur. Phys. J. C75 (2015) 476

[6] CMS Collaboration, Phys. Rev. D 92 (2015) 012004

[7] ATLAS and CMS Collaborations, JHEP 08 (2016) 045

[8] ATLAS Collaboration, Phys. Rev. D 97 (2018) 072003

[9] ATLAS Collaboration, Phys. Lett. B 784 (2018) 173

[10] ATLAS Collaboration, Phys. Rev. D 98 (2018) 052003

[11] ATLAS Collaboration, Phys. Lett. B 786 (2018) 59

[12] ATLAS Collaboration, Phys. Rev. D 98 (2018) 052003

[13] ATLAS Collaboration, Phys. Rev. D 97 (2018) 072016

[14] ATLAS Collaboration, ATLAS-CONF-2018-021, https://cds.cern.ch/record/2621794

[15] ATLAS Collaboration, Phys. Rev. Lett. 120 (2018) 211802

[16] ATLAS Collaboration, ATL-PHYS-PUB-2018-016

[17] ATLAS Collaboration, ATL-PHYS-PUB-2018-006

[18] ATLAS Collaboration, ATLAS-CONF-2018-026, 2018, lnuhttps://cds.cern.ch/record/2628763

[19] ATLAS Collaboration, arXiv:1808.09054 [hep-ex]

[20] ATLAS Collaboration, ATLAS-CONF-2018-018, https://cds.cern.ch/record/2621479

[21] ATLAS Collaboration, ATLAS-CONF-2018-028, https://cds.cern.ch/record/2628771

[22] ATLAS Collaboration, Phys. Lett. B 784 (2018) 345

[23] ATLAS Collaboration, Phys. Lett. B 786 (2018) 223

[24] ATLAS Collaboration, ATLAS-CONF-2018-018, https://cds.cern.ch/record/2621479

[25] ATLAS Collaboration, ATLAS-CONF-2018-028, https://cds.cern.ch/record/2628771

[26] ATLAS Collaboration, ATLAS-CONF-2018-031, 2018, https://cds.cern.ch/record/2629412 